\title{
PANCREATIC CARCINOMA: REVIEW OF LITERATURE
}

\author{
L. Veena Kumari ${ }^{1}$
}

\section{HOW TO CITE THIS ARTICLE:}

L. Veena Kumari. "Pancreatic Carcinoma: Review of Literature". Journal of Evolution of Medical and Dental Sciences 2015; Vol. 4, Issue 37, May 07; Page: 6517-6531, DOI: 10.14260/jemds/2015/945

\begin{abstract}
It is well known that the prognosis of pancreatic cancer is extremely poor, even when treated with radical surgery. The overall 5 year survival rate following surgical intervention is around $10 \%$.With the increasing use of CT scans for other reasons not related to pancreas, a variety of neoplastic and non-neoplastic lesions are increasingly encountered in clinical practice. The distinction of these lesions has significant therapeutic and prognostic implications. Regarding ductal carcinoma, key distinguishing features from chronic pancreatitis and a discussion of the concept of pancreatic intraepithelial neoplasia (PanIN) are included. Precursors, molecular carcinogenesis, risk factors and different morphological patterns of tumors arising from exocrine pancreas are discussed. Research on early detection is ongoing. Screening of people with a family history of hereditary pancreatitis plays an important role in the early detection of ductal carcinoma of pancreas.
\end{abstract}

KEYWORDS: pancreatic cancer, ductal adenocarcinoma, exocrine cancers.

INTRODUCTION: Pancreatic cancer arises when cells in the pancreas, a glandular organ behind the stomach, begin to multiply out of control and form a mass. These cancer cells have the ability to invade other parts of the body.(1) There are a number of types of pancreatic cancers. The most common pancreatic adenocarcinoma, accounts for about $85 \%$ of cases, and the term "pancreatic cancer" is sometimes used to refer only to that type. These adenocarcinomas start within the part of the pancreas which make digestive enzymes. 1-2\% of pancreatic cancers are neuroendocrine tumors arising from the hormone producing cells of the pancreas. These are less aggressive than adenocarcinomas.

Common signs and symptoms are yellow skin, abdominal or back pain, unexplained weight loss, light colored stools, dark urine and loss of appetite. The signs and symptoms are not specific to suspect pancreatic cancer. By the time of the diagnosis, pancreatic cancer has often spread to other parts of the body. $(2,3,4)$

Pancreatic cancer rarely occurs before the age of 40 and more than half of cases of pancreatic carcinoma occurs in those over 70.(2) Risk factors for pancreatic cancer include tobacco smoking, obesity, diabetes and certain rare genetic conditions.(2) About $25 \%$ of cases are linked to smoking(5) and $5-10 \%$ are linked to inherited genes.(2) Pancreatic cancer is usually diagnosed by a combination of medical imaging techniques such as ultrasound or computed tomography, blood tests, and examination of tissue samples (biopsy).(5,6)

The risk of developing pancreatic cancer is lower among non-smokers and people who maintain a healthy weight and limit their consumption of red or processed meat.(7) A smokers chance of developing the disease decreases if they stop smoking, and almost returns to that of the rest of the population after 20years. (4)

In 2012, pancreatic cancers of all types were the seventh most common cause of cancer deaths, resulting in 330,000 deaths globally.(4) In United States, pancreatic cancer is the fourth most common cause of deaths due to cancer.(8,9) The disease most often developed in the developed world, 
where about $70 \%$ of the new cases in 2012 originated.(4) Pancreatic adenocarcinoma typically has a very poor prognosis. After diagnosis, $25 \%$ of the people survive one year and $5 \%$ live for five years. $(4,10)$ For cancers diagnosed early, the five year survival rate rises to about $20 \% .{ }^{(11)}$ Neuroendocrine cancers have better outcomes; at five years from diagnosis, $65 \%$ of those diagnosed are living, though survival varies considerably depending on the type of tumor.(4)

TYPES: The organ, pancreas gets its name from the Greek pancreas, meaning "all flesh", the pancreas is, in fact, a complex lobulated organ, with distinct exocrine and endocrine compartments. The exocrine portion, which produces digestive enzymes, constitutes $80 \%$ to $85 \%$ of the pancreas. The endocrine portion is composed of about 1 million clusters of cells, the islets of Langerhans. The islet cells secrete insulin, glucagon and somatostatin and constitute only $1 \%$ to $2 \%$ of the organ.

The exocrine pancreas is composed of acinar cells, which produce enzymes needed for digestion. The acinar cells are pyramidally shaped epithelial cells that are radially oriented around a central lumen.

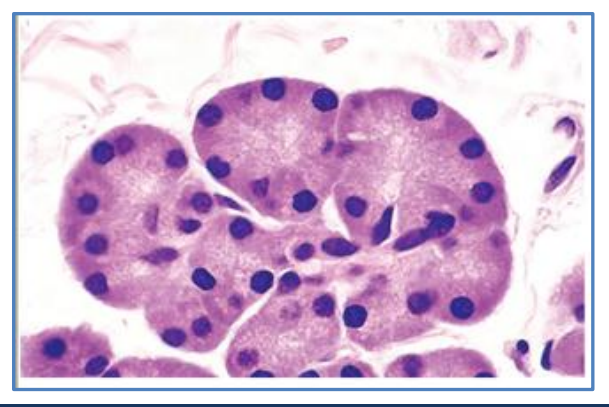

Fig. 1: Pancreatic acini, showing the radial

orientation of the pyramidal exocrine acinar cells

$99 \%$ of cases, vast majority of cases occur in the exocrine component. Small minority of cancers arise in the hormone producing endocrine tissue of the pancreas. Both groups occur mainly in people over 40, and are slightly more common in men and some rare subtypes mainly occur in women or children.

EXOCRINE CANCERS: The most common type representing about $85 \%$ of all pancreatic cancers ${ }^{(2)}$ is pancreatic adenocarcinoma (invasive and ductal). These arises from the pancreatic ductal epithelium - represents less than $10 \%$ of the pancreas by cell volume. This cancer arises in the ducts that carry certain hormones and enzymes away from the pancreas. About $60-70 \%$ of adenocarcinomas occur in the head of the pancreas. ${ }^{(2)}$

The next most common type, acinar cell carcinoma of the pancreas, arises in the clusters of the cells that produces these enzymes, and represents $5 \%$ of exocrine cancers.

Cystadenocarcinomas account for $1 \%$ of pancreatic cancers and they have a better prognosis than the other exocrine types.(7)

Pancreatoblastoma is a rare form, mostly occurring in childhood, with a relatively good prognosis. Other exocrine cancers include adenosquamous carcinomas, signet ring cell carcinomas, hepatoid carcinomas, colloid carcinomas, undifferentiated carcinomas and undifferentiated 
carcinomas with osteoclast like giant cells. Solid pseudopapillary tumor is a rare low grade neoplasm that mainly affects younger women, and generally has a good prognosis. $(2,12)$

Pancreatic mucinous cystic neoplasms are a group of pancreatic tumors that have varying malignant potential. They are being detected at a greatly increased rate as CT scans become more powerful and common.(9) They are seen in younger age group and predominate in women. They are characterized by the formation of large multilocular or on rare cases, unilocular cysts lined by tall, mucin producing, often forming papillae.(12,13,14) Most are found in the body and tail rather than in the head. Calcification in the wall is a common finding. Aspiration of the fluid can be useful in the differential diagnosis. These pancreatic tumors can be divided into benign, borderline and malignant. The diagnosis of malignancy is based on the presence of invasion of the wall by neoplastic glands or frank neoplasia of the superficial component. All of these mucinous cystomas are potentially malignant, a total excision should be carried out whenever possible.

NEUROENDOCRINE TUMORS: The small minority of tumors that arise elsewhere in the pancreas are mainly pancreatic neuroendocrine tumors (PanNETs).(15) Neuroendocrine tumors are a diverse group of benign or malignant tumors that arise from the body's neuroendocrine cells, which are responsible for integrating the nerves and endocrine systems. NETs can start in most organs of the body including the pancreas. Pan NETs are grouped into 'functioning' and 'non-functioning' types depending upon the degree to which they produce hormones. The functioning types secrete hormones such as insulin, gastrin and glucagon into the blood stream, often in large quantities, giving rise to serious symptoms such as low blood sugar, and also favoring relatively early detection. The most common functioning Pan NETs are insulinomas and gastrinomas, named after the hormones they secrete. The nonfunctioning tumors do not secrete hormones in sufficient quantity to produce clinical symptoms. For this reason, non-functioning PanNETs are often diagnosed only after they metastasize.(16)

The classification of PanNETs is complex,(17) PanNETs are sometimes called "islet cell cancers" $(8)$ even though it is now known that they do not actually arise from islet cells as previously thought. (18)

Risk factors(2,3,4): These include.

Age, gender and race: The risk of developing cancer increases with age. Most cases occur after age,(4) uncommon before the age of 40 . The disease is slightly more common in men than women. The incidence is increasing in women.(19) In United States the incidence is 1.5 times more common in African Americans, though the incidence in Africa is low.(4) It is more common in blacks than in whites and is slightly more common in individuals of Ashkenazi Jewish descent.

Cigarette smoking is the best established avoidable risk factor for pancreatic cancer, approximately doubling risk among long term smokers, the risk increases with the number of cigarettes smoked and years of smoking. The risk declines slowly after smoking cessation, taking some 20years to return to almost that of non - smokers. ${ }^{(20)}$

Obesity - a BMI greater than 35 increases relative risk by about half.(3)

Family history: 5 - 10\% of pancreatic cancers have an inherited component, where people have a family history of pancreatic cancer.(2) The risk escalates greatly if more than one first degree relative 
has the disease and more modestly if they developed it before the age of 50.(6) Hereditary pancreatitis gives a greatly increased lifetime risk of pancreatic cancer of $30-40 \%$ to the age of $70 .(5)$

Pancreatic cancer has been associated with the following other rare hereditary syndromes: 1) familial breast cancer with germline mutation of BRCA2. 2) Peutz - Jeghers syndrome due to mutations in the STK11 tumor suppressor gene (very rare, but a very strong risk factor). 3) dysplastic nevus syndrome (or familial atypical multiple mole and melanoma syndrome, FAMMM-PC) due to mutations in the CDKN2A tumor suppressor gene. 4) autosomal recessive ataxia - telengiectasia and autosomal dominantly inherited mutations in the BRCA2 gene. 5) hereditary non polyposis colon cancer (Lynch syndrome). 6) familial adenomatous polyposis and 7) hereditary pancreatitis with germline mutations in the cationic trypsinogen gene. PanNETs have been associated with 8) multiple endocrine neoplasia type 1 (MEN 1) and 9) von Hippel Lindau syndromes.(2,5,6)

\begin{tabular}{|c|c|c|c|}
\hline Disorder & $\begin{array}{l}\text { Gene (Chromosome } \\
\text { Location) }\end{array}$ & $\begin{array}{l}\text { Increased Risk of Pancreatic } \\
\text { Cancer fold }\end{array}$ & $\begin{array}{l}\text { Risk of Pancreatic Cancer by } \\
\text { Age } 70(\%)\end{array}$ \\
\hline Hereditary breast and ovarian cancer & $B R C A 2(13 q 12-q 13)$ & $4-10$ & 5 \\
\hline $\begin{array}{l}\text { Familial atypical multiple-mole melanoma } \\
\text { syndrome }\end{array}$ & p16/CDKN2A (9p21) & $20-35$ & $10-17$ \\
\hline $\begin{array}{l}\text { Strong family history ( } 3 \text { or more relatives } \\
\text { with pancreatic cancer) }\end{array}$ & Unknown & $14-32$ & $8-16$ \\
\hline Hereditary pancreatitis & $\begin{array}{l}\text { PRSS1 }(7 q 35) \text { and } \\
\text { SPINK1 }\end{array}$ & $50-80$ & $25-40$ \\
\hline Peutz-Jeghers syndrome & LKB) (19p13) & 130 & $30-60$ \\
\hline
\end{tabular}

Chronic pancreatitis appears to almost triple risk, and as with diabetes, new onset pancreatitis may be a symptom of tumor.(5) The risk of pancreatic cancer in individuals with familial pancreatitis is particularly high. ${ }^{(5,21)}$ It has been suggested that variations in pancreaticobiliary ductal anatomy may be related to the incidence of carcinoma, in view of the fact that, lack of a common channel has been found at autopsy to be associated with microscopic abnormality of the ductal epithelium.

Diabetes mellitus is a risk factor for pancreatic cancer and new onset diabetes may also be an early sign of the disease. People who have been diagnosed with type 2 diabetes for longer than ten years may have a $50 \%$ increased risk, as compared with non diabetics.(5) Over production of islet amyloid polypeptide by beta cells may contribute to this complication.(22)

Specific types of food have not been clearly shown to increase the risk of pancreatic cancer.(2) There is some evidence of slightly increased risk with factors include processed meat, red meat and meat cooked at very high temperature (eg: by frying, broiling or barbecuing).(23,24) Fruits, vegetables, fibers, folate and vit.C may be protective.

Alcohol: drinking alcohol excessively a major cause of chronic pancreatitis which in turn predisposes to pancreatic cancer. Cases of pancreatic carcinoma have been reported in the past in workers exposed to $\beta$ - naphthylamine or benzidine.(25) 


\section{Molecular Carcinogenesis and Precursors:}

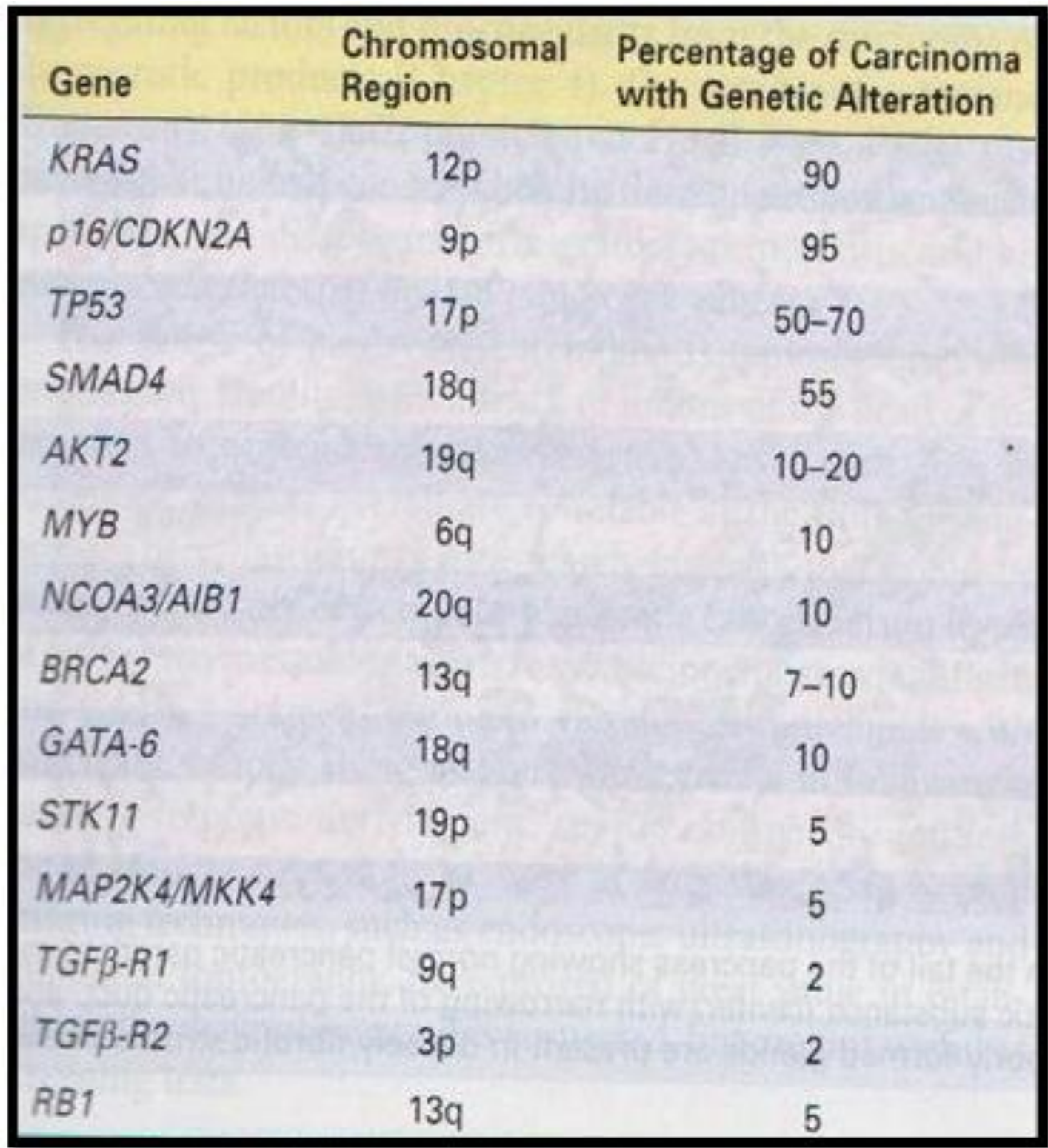

Table 2: Molecular alterations in invasive pancreatic adenocarcinoma

These cancers are thought to arise from several types of precancerous lesions within the pancreas. But these lesions do not always progress to cancer, and the increased numbers detected as a by product of the increasing use of CT scans for other reasons not related to pancreas. Apart from pancreatic serous cystadenomas (SCNs), which are almost always benign, three types of precancerous lesions are recognized.

The First is the pancreatic intra epithelial neoplasia. These are microscopic abnormalities in the pancreas, which are often found in autopsies of people with no diagnosed cancer. These may progress from low to high grade and then to a tumor. More than $90 \%$ of cases at all grades carry a 


\section{REVIEW ARTICLE}

faulty KRAS gene, while in grades 2 and 3 damage to three further genes - CDKN2A ( $\left.\mathrm{p}^{16}\right), \mathrm{p}^{53}$ and $\mathrm{SMAD}^{4}$ are increasingly often found.(2)

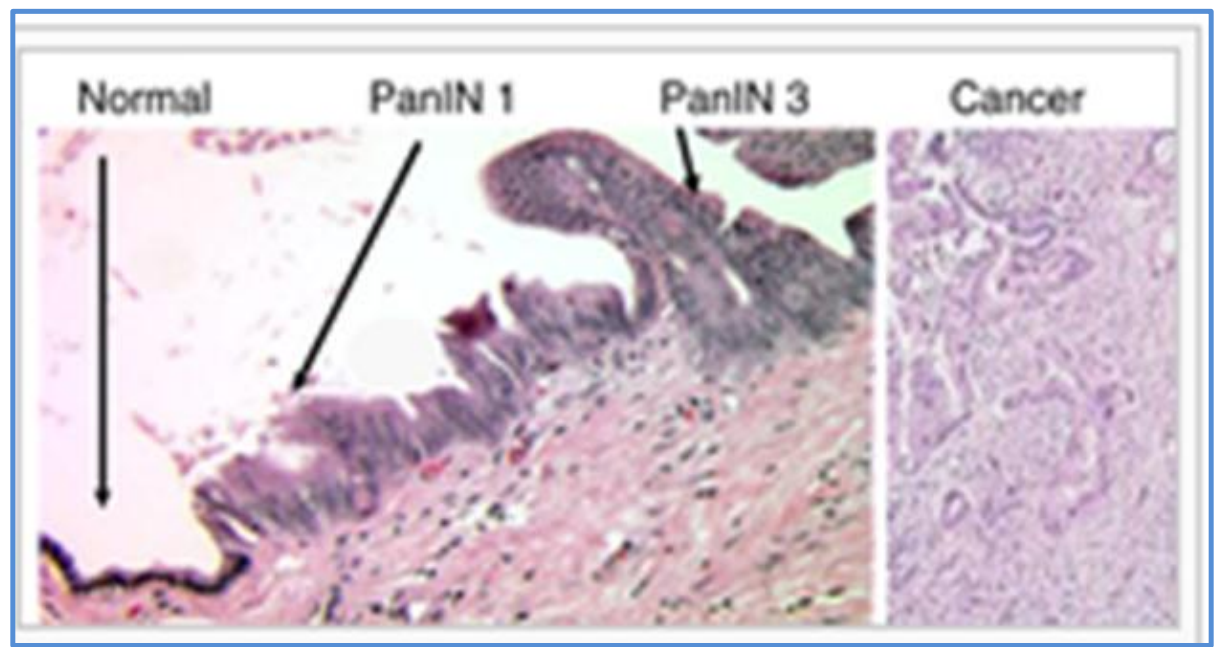

Fig. 2: Micrographs of normal pancreas, pancreatic intraepithelial neoplasia (precursors to pancreatic carcinoma) and pancreatic carcinoma. H \& E stain.

Secondly, intraductal papillary mucinous neoplasms (IPMNs) are macroscopic lesions, which occur in about $2 \%$ of all adults, rising to about $10 \%$ by age 70 , and have about a $25 \%$ risk of developing into invasive cancer. The gross and microscopic appearance of these tumors is dependent upon the interplay of two factors: epithelial proliferation and mucinous secretion. When the former predominates, the result is a multicentric involvement of major ducts by a predominating papillary lesion, sometimes combined with cribriform features and varying degrees of cytological atypia. This atypia can be detected in cytologic preparations from pancreatic juice. When mucinous secretion predominates the lesion shows cystic formation lined by mucus secreting columnar epithelium.

They also very often have KRAS gene mutations, in about 40 - 65\% of cases and in the GNASGs alpha subunit and RNF43, affecting the wnt signaling pathway.(2) Even if removed surgically, there remains a considerably increased risk of pancreatic cancer developing subsequently. ${ }^{(5)}$

The last type, pancreatic mucinous cystic neoplasms (MCNs) mainly occur in women, and may remain benign or progress to cancer.(11) Nearly all invasive (colloid) carcinomas arise from in - situ papillary neoplasms. If they become large, cause symptoms or have suspicious features, they can usually be successfully removed by surgery.(5) IPMN, is as a rule, clinically detectable, grossly visible identifiable mucin, and well-formed papillae, whereas the reverse is true for PanIN.(26)

The genetic events found in ductal adenocarcinoma have been well characterized, and complete exome sequencing has been done for the common types of tumor. Four genes have been found to be mutated in the majority of adenocarcinomas. KRAS (in 95\% of cases), CDKN2A (also in 95\%), TP53 (75\%), and SMADA (55\%). The last of these are associated with a poor prognosis.(5) SW1/SNF mutations/ deletions occur in about $10-15 \%$ of the adenocarcinomas.(2) The genetic alterations in several other types of pancreatic cancer and precancerous lesions have also been researched.(5)

Molecular alterations in pancreatic carcinogenesis are summarized in table 2. 
KRAS: The KRAS gene (chromosome 12p) is the most frequently altered oncogene in pancreatic cancer. This oncogene is activated by point mutations in $80 \%$ to $90 \%$ of cases. These point mutations impair the intrinsic guanosine triphosphatase activity of the K-ras protein, resulting in a protein that is constitutively active.

CDKN2A(p16): This gene (chromosome 9p) is inactivated in 95\% of the cases, making $\mathrm{p}^{16} / \mathrm{CDKN} 2 \mathrm{~A}$ the most frequently inactivated tumor suppressor gene in pancreatic cancer.(17)

SMAD4: The SMAD4 tumor suppressor gene (chromosome 18q) is inactivated in 55\% of pancreatic cancers.(10)

P53: Inactivation of the $\mathrm{P}^{53}$ tumor suppressor gene (chromosome17p) is seen in $50 \%$ to $70 \%$ of pancreatic cancers.

PanNETs: The genes often found mutated in PanNETs are different from those in pancreatic adenocarcinomas.(12) For example KRAS mutation is normally absent. Instead, hereditary MEN1 gene mutations give rise to MEN1 syndrome, in which primary tumors occur in two or more endocrine glands. About 40 - 70\% of people born with a MEN1 mutation eventually develop a PanNET.(27) Other genes that are frequently mutated include DAXX, mTOR and ATRX.(18)

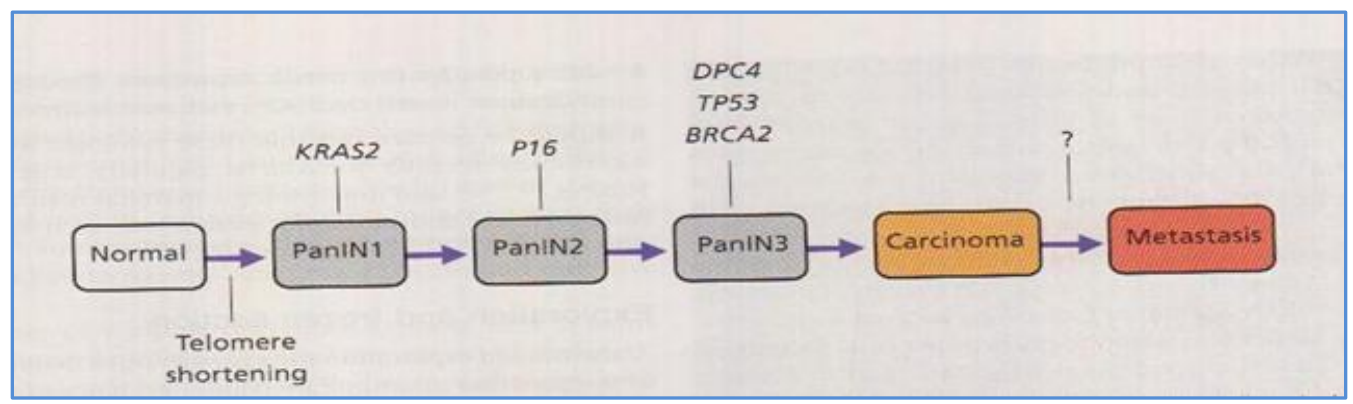

Fig. 3: Genetic progression model of pancreatic carcinogenesis.

The progression from histologically normal epithelium to low grade pancreatic intraepithelial neoplasia (PanIN1 and PanIN2), to high grade PanIN3, to invasive carcinoma (left to right) is associated with the specific genetic alterations. On the basis of their temporal appearance in this progression model, the molecular abnormalities can be classified as early (KRAS2 mutation, telomere shortening), intermediate (P16/CDKN2A loss), or late (mutations of DPC4/SMAD4, TP53, BRCA2).

Several techniques have been used with various degrees of success to detect pancreatic carcinoma at an early stage.(28) These include CT scan, nuclear magnetic resonance, celiac angiography, sonography, endoscopic retrograde cholangiopancreatography (ERCP), selenom ethionine scan, duodenal aspiration and serum tests.(29) The latter use monoclonal antibodies against various cancer related antigens. Those measuring levels of Span -1 and CA 19-9 antigen seem more effective than those measuring carcinoembryogenic antigen (CEA) or other markers.(30) Computed tomography is more sensitive $(90 \%$ for tumors $>2 \mathrm{~cm})$, high resolution techniques used to determine respectability.(31) 
Endoscopic ultrasound (EUS) is complementary. It has a sensitivity of $90 \%-95 \%$, is superior at detecting small lesions and through its coupling with fine needle aspiration allows for direct tissue sampling with a specificity approach (100\%).(31,32)

Pancreatic Ductal Adenocarcinoma: Location and gross features: Pancreatic ductal carcinoma is located in the head of the pancreas in $2 / 3^{\text {rds }}$ of patients and in the body or tail in the other third. Multiple tumors are found in about $20 \%$ of the cases. Most tumors are poorly deleniated and firm, with a yellowish gray cut surface. Rarely the tumor undergoes massive cystic degeneration.(13) The duodenal wall is invaded by direct extension in one fourth of the tumors arising from the pancreatic head. The involved pancreatic ducts frequently become greatly dilated and plugged with necrotic tumor. Extra pancreatic extension is common, in which case the origin of the tumor is difficult to determine. Cubilla and Fitzgerald(33) found that one third of the cases clinically regarded as pancreatic carcinomas were not of pancreatic origin but rather of duodenal (ampullary), retroperitoneal, or metastatic nature. Of 28 patients in whom the carcinoma was grossly located in the area of the head of the pancreas, an origin from the pancreatic ducts could be proved in only 14 . In 5 , the site of origin could not be determined; in the others, the tumors originated in the ampulla, bile ducts or duodenum.

The non-neoplastic pancreas distal to the tumor may show extensive atrophy, chronic inflammation, fibrosis and ductal dilatation. They may also show precursor lesions, PanINs especially in familial cases.(34)

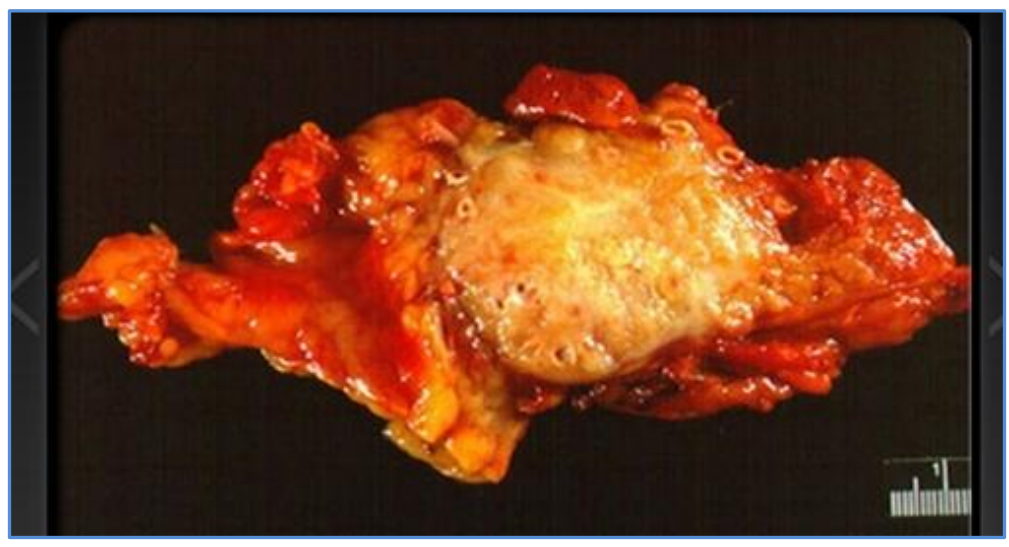

Fig. 4: Gross appearance of invasive ductal carcinoma. Note the yellowish gray white cut surface.

Microscopic Features: Pancreatic ductal adencarcinoma are graded microscopically into well differentiated, moderately differentiated and poorly differentiated. In well differentiated tumors under low power magnification the glands are well formed, have a large lumen and are lined by one or more layers of cuboidal epithelium, but the glands are irregular in shape and distribution with prominent concentric desmoplastic stroma surrounding them. High power magnification will reveal the malignant features like marked nuclear pleomorphism, loss of polarity, prominent nucleoli and mitotic figures. These features are typical of pancreaticobiliary origin.(35) Perineural invasion, which is present in $90 \%$ of the cases, constitutes an additional important diagnostic sign. 


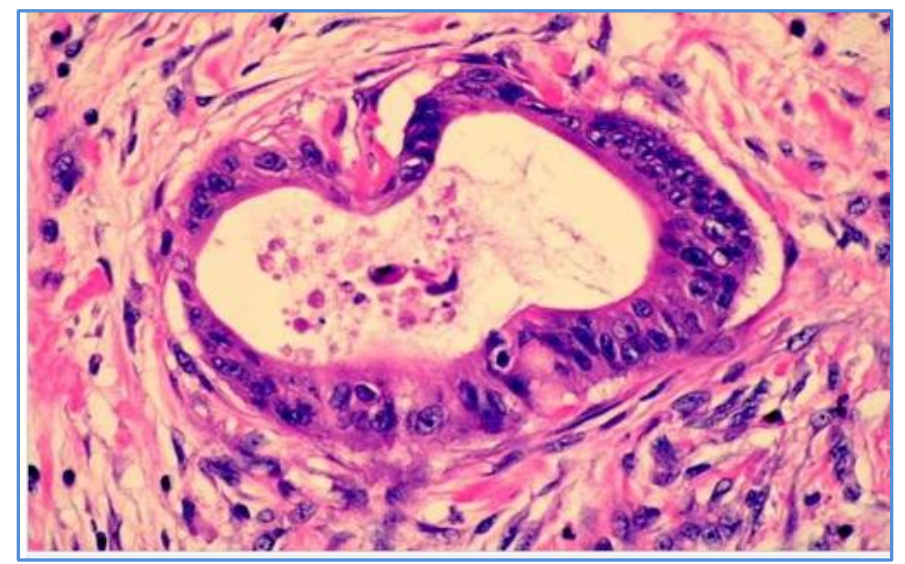

Fig. 5: High power view of moderately differentiated invasive adenocarcinoma shows loss of polarity, nuclear stratification, nuclear hyperchromasia and prominent nucleoli. Note the desmoplastic stroma around the gland

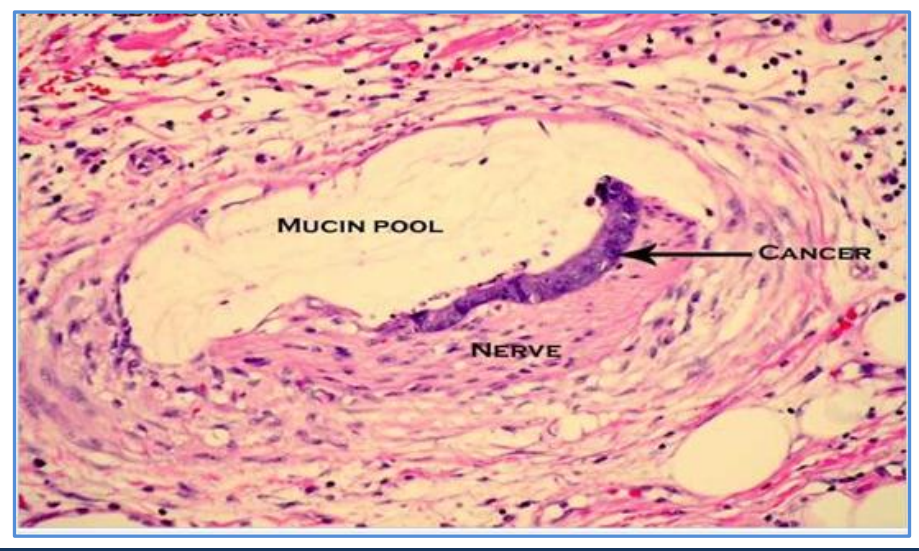

Fig. 6: Showing perineural invasion of pancreatic ductal carcinoma supports the diagnosis of malignancy in well differentiated tumors.

However there are two caveats: benign epithelial inclusions have been observed in pancreatic nerves,(36) and perineural extension of islet cells can occur in chronic pancreatitis.(37) Invasion of blood vessels, particularly veins is seen in half of the tumors. But a careful autopsy study by Wachtil Miller(38) showed that this finding is not unusual in chronic pancreatitis with parenchymal atrophy and no tumor. The most important entity in the differential diagnosis of pancreatic adenocarcinoma is chronic pancreatitis. Pseudo tumoral pancreatitis refers to cases in which a mass lesion is identified. Biopsy in these can adenocarcinoma may induce chronic pancreatitis in the surrounding pancreas and chronic pancreatitis itself a risk factor for adenocarcinoma. As such, a negative biopsy does not exclude malignancy. Pancreatic resection for complications of chronic pancreatitis represents an acceptable treatment option in some patients. $(39,40)$

Autoimmune pancreatitis: (also termed lymphoplasmacytic sclerosing, chronic sclerosing and nonalcoholic duct destructive chronic pancreatitis) deserves special mention.(41,42) The term autoimmune pancreatitis is currently preferred since clinical, serologic, histologic, and immunohistochemical 


\section{REVIEW ARTICLE}

findings suggest an autoimmune mechanism, and it is often associated with other autoimmune conditions including primary sclerosing cholangitis, sjogren syndrome and inflammatory bowel disease. This potentially steroid responsive condition is usually treated by whipple procedure since patients typically present with signs and symptoms simulating malignancy (eg: obstructive jaundice due to stenosis of the common bile duct, mass lesion on CT). Microscopic features include dense periductal lymphoplasmacytic inflammation, fibrosis and obliterative venulitis. Other features may include arteritis and so called "granulocytic epithelial lesions" (granulocytic periductal and ductal inflammation with or without rupture and destruction of the ductal epithelium). IgG ${ }^{4}$ staining in plasma cells has been used by some to assist in this diagnosis. However, stainig can be seen in chronic pancreatitis from other causes and rarely in pancreatitis surrounding ductal adenocarcinoma. ${ }^{(41,42)}$

PanIN represents the recognized precursor to most invasive ductal adenocarcinoma. Four lesions are recognized, believed to represent points along a continuum of neoplastic progression. PanIN - 1A consists of ducts lined by flat, basal appearing columnar mucinous epithelium, generally resembling gastric mucinous epithelium. In PanIN - 1B, similar appearing epithelium is thrown up into small tufts. PanIN - 2 lesions exhibit distinct nuclear abnormalities including elongation, hyperchromasia and stratification. PanIN - 3 is not distinguished from carcinoma - in situ. Lesions frequently demonstrate micropapillary architecture and reveal severe nuclear abnormalities including "rounding - up" of nuclei, prominent nucleoli and prominent mitotic figures. Rare variants of PanIN with intestinal, oncocytic, and foamy features have been described in the setting of mucinous carcinoma, intraductal oncocytic papillary carcinoma and foamy gland carcinoma respectively. ${ }^{(8,10)}$ While PanIN - 1 is a frequently encountered incidental finding, high grade PanIN are frequently seen adjacent to invasive carcinoma. Similar high grade preinvasive lesions are described in some patients with long standing chronic pancreatitis. PanIN have shown to have some genetic abnormalities with ductal adenocarcinomas (eg; KRAS activating mutations and loss of $\mathrm{p}^{53,} \mathrm{p}^{16}$ and $\mathrm{DPC}^{4}$ function) and patients with high grade lesions have progressed to invasive carcinoma on follow up.

Carcinoma in situ (High grade pancreatic intraepithelial neoplasia) is found in the duct epithelium adjacent to the carcinoma in about 20 - 30\% of the cases, sometimes at a distance from the main tumor mass and even at the point of surgical resection.(43,44) Carcinoma in situ and atypical hyperplasia (now grouped under the term pancreatic intraepithelial neoplasia or PanIN) are regarded as precursors of invasive ductal adenocarcinoma.(45) This belief is supported by the realization that some of these lesions have been identified in pancreas years before the development of invasive carcinoma, the fact that molecular genetic analysis has revealed that they frequently harbor many of the same genetic alterations present in them of telomere shortening.(20)

The lobular tissue may be completely destroyed because of ductal occlusion by the invasive carcinoma. The islet tissue is usually well preserved, resulting in an appearance designated as insular pancreas. However both atrophic and hypertrophic changes can occur in the islets. Most commonly, destruction of a variable amount of islet tissue mass results in a subclinical or overt diabetic picture. Rarely, hypertrophy of the islets occurs distal to a ductal carcinoma may produce hypoglycemia. Gambill(33) found significant pancreatitis in 26 (10\%) of 255 patients with pancreatic or ampullary carcinoma. The presence of pancreatitis resultrd in a considerable delay in the diagnosis of carcinoma. 
Other microscopic types: other uncommon variants of adenocarcinoma include adenosquamous carcinoma, oncocytic carcinoma, clear cell carcinoma, hepatoid carcinoma, signet ring carcinoma, basaloid carcinoma, intestinal type carcinomas and mucinous carcinoma. Mucinous (colloid) carcinoma, which may result in pseudomyxoma peritonei( ${ }^{(34)}$ has been said to result from the combined effect of the production of MUC2 (a gel forming mucin), altered cell polarity and abnormal cell stroma interaction.(21) A few reported cases of pure squamous cell carcinoma of pancreas may represent a variant of adenosquamous carcinoma in which the squamous component has overrun the glandular elements, this tumor type can be associated with hypercalcemia.(24)

Adsay et al(46) have described a subtype of ductal carcinoma with a foamy gland pattern having a deceptively benign appearance and Wilenz et al(47) have identified a medullary carcinoma characterized by a solid, sometimes lymphoepithelioma like pattern of growth, usually wild type (non-mutated) KRAS, and occasional multicentricity.

Microadenocarcinoma represents a pattern of growth associated with an aggressive clinical course and not a distinctive morphologic entity ${ }^{(39)}$ according to the study conducted by Lonardo $\mathrm{F}$ et al.

Anaplastic carcinoma: It is a pleomorphic, sarcomatoid, undifferentiated variant of duct derived carcinoma with aggressive behavior.(40) They comprise $7 \%$ of all non-endocrine pancreatic malignancies. Most involve the body or tail of the pancreas rather than the head. Majority of the patients are above the age of 50 with male predilection.

\section{Three Morphological Patterns are Recognized sometimes in Combination.(48):}

1. Pleomorphic carcinoma contain a large number of bizarre, multinucleated tumor cells. These can be confused with amelanotic melanoma, hepatocellular carcinoma and sometimes of sarcoma. Both lymph born and hematogenous metastases are very common.

2. Tumors that are largely composed of spindle shaped cells and can easily be confused with sarcoma.

3. Tumors that are composed of small, monotonous round cells growing in a solid fashion. Some of these tumors exhibit features of neuroendocrine differentiation.

Any of these varieties can be accompanied by areas of ductal adenocarcinoma or a mucinous cystic neoplasm. Immunohistochemically, stains for keratin, EMA and CEA are positive in the obvious epithelial component and sometimes also in the sarcoma like areas. The prognosis in all these varieties is extremely poor.

Giant cell tumor of the pancreas is a different morphological pattern and has better prognosis. Grossly usually large and hemorrhagic. Microscopically the tumor has a dual population: relatively uniform spindle cells of mesenchymal appearance and atypical cytologic features alternate with multinucleated giant cells having an appearance and histochemical profile indistinguishable from that of normal osteoclasts.(49,50,51) The nuclei of these osteoclast like cells are uniformly small and mitosis are absent. Bizarre giant cells with atypical nuclei are not found.

Acinar cell carcinoma: usually occurs in adults but may also develop in children. Grossly presents as a relatively well circumscribed fleshy mass averaging $11 \mathrm{~cm}$ in greatest diameter, sometimes with extensive hemorrhage and necrosis. Microscopically it is a cellular neoplasm that lacks desmoplastic stroma. The pattern of growth may be solid, trabecular, glandular or papillary or may produce acini simulating normal pancreas.(52,53) 
The nuclei are round to oval with only mild pleomorphism, single prominent nucleoli and variable mitotic activity. The cytoplasm tends to be abundant, eosinophilic and granular, but in solid tumors it may be scanty. PAS positive, diastase resistant cytoplasmic zymogen granules are common, and the butyrase esterase histochemical stain for lipase activity is usually positive. There is immunoreactivity for trypsin, lipase and less commonly chymotrypsin and amylase.(54) Recently an antibody has been deacribed known as anti - BCL10 (clone 331.1), which promises to be a very sensitive and specific marker of acinal cells and their tumors.

Several variants of acinar cell tumors have been described: 1) acinar cell cystadenocarcinoma 2) mixed acinar endocrine tumor 3) mixed acinar ductal carcinoma and 4) a rare tumor combining ductal, acinar and islet cell components.

CONCLUSION: Worldwide efforts on many levels are underway to understand pancreatic cancer, but progress has been slow, particularly into understanding the disease causes. The nature of the changes that lead to the disease are being intensely investigated, such as the roles played by genes such as KRAS and $\mathrm{p}^{53 .(21)}$ A key question is the timing of events as the disease develops and progresses particularly the role of diabetes and how and when the disease spreads.

Research on early detection is ongoing.(31,32) For instance, the European Registry of Hereditary Pancreatitis and Familial Pancreatic Cancer (EUROPAC) trial is aiming to determine whether regular screening is appropriate for people with a family history of the disease or who have hereditary pancreatitis. The knowledge that new onset of diabetes can be an early sign of the disease could facilitate timely diagnosis and prevention if a workable screening stategy can be developed.

\section{REFERENCES:}

1. "What is cancer? Defining cancer". National cancer Institute. National Institute of Health. 7 march 2014. Retrieved 5 December 2014.

2. Ryan DP, HongTS, Bardeesy N (September 2014)."Pancreatic adenocarcinoma": N. Engl.J. Med 371(11) $1039-49$.

3. Bond Smith G, Banga N, Hammond TM, Imber CJ (2013) "Pancreatic adenocarcinoma". BMJ (Clinical research ed.)344. e2476.

4. World cancer Report 2014. World Health Organization. 2014. Chapter 5.7 ISBN 92 - 832 - 0429 - 8.

5. Wolfgang CL, Heman JM, Laheru DA, Klein AP, Erdek MA et al. (September 2013) "Recent progress in Pancreatic cancer". CA. a cancer. Journal for clinicians. 63(5): 318 - 48.

6. Vincent A, Herman J, Schulick R et al (August 2011)."Pancreatic cancer". Lancet 378 (9791): $607-20$.

7. Tobias JS, Hochhauser D (2010). Cancer and its management (6th ed) PP.276 - 7. ISBN 978 - 1 $-1187-1325-9$.

8. The medical subject Headings indexing system refers to "islet cell carcinoma", which is subdivided into gastrinoma, glucagonoma, somatostatinoma and VIPoma. See: $2014 \mathrm{MeSH}$ tree at "Pancreatic Neoplasms" \{C04: 588: 322.475\} 16 Oct 2014.

9. Farrell JJ, Fernandez - del Castillo C (june2013), "Pancreatic cystic neoplasms: management and unanswered questions". Gasroenterology 144(6): 1303 - 15. 
10. Caldas $\mathrm{C}$ et al: Frequent somatic mutations and homozygous deletion of the $\mathrm{p}^{16}$ (MTS 1) gene in pancreatic adenocarcinoma. Nat Genet 8: 27, 1994.

11. Delper Y, Hanoun N, Lulka H, Sicard F, Selves J et al (2011). Genetic and epigenetic alterations in pancreatic carcinogenesis. Curr Genomics 12(1): 15 - 24.

12. Lewis MA, yao. JC."Molecular Pathology and genetics of gastrointestinal neuroendocrine tumors". Current opinion in Endocrinology and Diabetes and obesity 21(1): 22 - 7.

13. Kosmahl M, Pauser U, Analauf M, Kloppel G. Pancreatic ductal carcinoma with cystic features. Neither rare nor uniform. Mod. Pathol 2005, 18: 1157 - 1164.

14. Albores - Saavedra J, gould ED, Angeles - Angeles et al, Cystic tumors of the pancreas. Pathol. Annu 1990, 25 (Pt2): 1950.

15. Wilentz RE, Goggins. M.et al. Genetic immunohistochemical, and clinical features of medullay carcinoma of the pancreas. A newly described and characterized entity. Am.j. Pathol. 2000, 15: $1641-1651$.

16. Zamboni G, Scarpa A, et al. mucinous cystic tumors of the pancreas: clinicopathological features, prognosis and relationship to other mucinous cystic tumors. Am. J. Surg. Pathol 1999; 23: 410 422.

17. Jones $\mathrm{S}$ et al: Core signaling pathways in human pancreatic cancers revealed by global analysis. Science 321: 1801, 2008.

18. Burns WR, Edil BH (March 2012) "Neuroendocrine pancreatic tumors". Guidelines for management and update. Current treatment options in oncology 13(1): 24 - 34.

19. Warshaw AL, Fernandez - del Castillo C. Pancreatic carcinoma. N. Engl. J. Med. 1992. 326: 455 465.

20. Van Heek NT, Meeker AK, Kern SE, yeo CJ. Et al. Telomere shortening is nearly universal in pancreatic intraepithelial neoplasia. Am. J. Pathol 2002, 161: 1541 - 1547.

21. Gambell EE. Pancreatitis associated with pancreatic carcinoma. A study of 26 cases. Mayo clin.Proc 1971, 46: 173 - 177.

22. Permert J. Larson. J, Westernmark GT et al. Islet amyloid polypepide in patients with pancreatic cancer and Diabetes. N. Engl. J. Med: 1994; 330: 313 - 318.

23. Adsay NV, Merati K et al. Pathogenesis of colloid (pure mucinous) carcinoma of exocrine organs. Am J. Surg. Pathol 2003, 27: 571 - 578.

24. Brayko CM, Doll DC. Squamous cell carcinoma of the pancreas associated with hypercalcemia. Gastroenterology 1982, 83: 1297 - 1299.

25. Longnecker DS, Pathology and pathogenesis of diseases of the pancreas. Am. J. Pathol. 1982. 107: 103 - 121.

26. Hruban RH, Takaori $\mathrm{K}$ et al. An illustrated consensus on the classification of pancreatic intraepithelial neoplasia and intraductal papillary mucinous neoplasms. Am. J. Surg. Pathol.2004. 28: 977 - 987.

27. Thakker RV, Newey PJ et al. (September 2012) "clinical practice guidelines for multiple endocrine neoplasia type 1 (MEN1)". The journal of clinical Endocrinology and Metabolism 97 (9): $2990-3011$.

28. Van Riel JMGH, Giaccone G, Pinede HM. Pancreaticobiliary cancer: the future aspects of medical oncology: Ann.oncol: 2000, 10: s296 - s299. 
29. La Rosa S, Franze F et al. The monoclonal and anti Bcl - 10 antibody is sensitive and specific marker of pancreatic acinar cell carcinoma and pancreatic metaplasia. Virchows. Arch 2009, 454: 133 - 442 .

30. Gattani AM, Mandeli J, Bruckner HW. Tumor markers in patients with pancreatic carcinoma. Cancer 1996, 78; $57-62$.

31. Tamm E, Charnsangaveg C. Pancreatic cancer. Current concepts in imaging for diagnosis \& staging. Cancer J. 2001; 7: 298 - 311.

32. Rafique A, Freeman S, Carroll N. A clinical algorithm for the assessment of pancreatic lesions: utilization of 16 - and 64 - section multidetector CT \& endoscopic ultrasound. Clin. Radiol. 2007; 62: 1142 - 1152.

33. Cubilla AL, Fitzgerald PJ. Morphological patterns of primary nonendocrine human pancreas carcinoma. Cancer Res. 1975, 35; 2234 - 2248.

34. Shi C, Klein AP, Goggins M, Canto M et al. Increased prevalence of precyrsor lesions in familial pancreatic cancer patients. Clin. Cancer Res. 2009, 15; 1737 - 1743.

35. Longnecker DS, Terhune PG. The case for parallel classification of biliary tract and pancreatic neoplasms. Mod. Pathol 1996; 9: 828 - 837.

36. Costa J, Benign epithelial inclusions in pancreatic nerves. Am J. J. clin. Pathol. 1977. 67: 306 307.

37. Bartow S, Mukai K, Rosai J. Pseudoneoplastic proliferation of endocrine cells in pancreatic fibrosis. Cancer 1981, 47: 2627 - 2633.

38. Wachtel MS, Miller EJ, Focal changes of chronic pancreatitis and duct arteriovenous relationships: avoiding a diagnostic pitfalls, Am. J. Surg. Pathol. 2005, 29: 1521 - 1523.

39. Abraham SC, Wilentz RE, et al. Pancreatico duodenectomy (whipple resections) in patients without malignancy. Are they all "chronic Pancreatitis". Am. J. Surg. Pathol. 2003; 27: 110 - 120.

40. Proca DM, Ellison EC, Hibbut D, et al. Major pancreatic resections for chronic pancreatitis. Arch Pathol. Lab Med. 2001; 125: 1051 - 1054.

41. Deshpande V, Chicano S, et al. Autoimmune pancreatitis: A systemic immune complex mediated disease. Am. J Surg Pathol. 2006; 30: 1537 - 1545.

42. Kojima M, Sipos B, Klappa W, et al. Autoimmune pancreatitis: Frequency Ig G4 expression and clonality of T B cells. Am. J. Surg. Pathol. 2007: 31: 521 - 528.

43. Edis AJ, Kieman PD, Taylor WF: attempted curative resection of ductal carcinoma of the pancreas. Review of Mayo. Clin. Proc - 1980, 55: 531 - 540.

44. Tryka AF, Brooks JR, Histopathology in the evaluation of total pancreatectomy for ductal carcinoma. Ann.Surg. 1979, 190: 373 - 381.

45. Andea A, Sarkar F, Adsay VN. Clinicopathological correlates of pancreatic intraepithelial neoplasia: a comparative analysis of 2 cases with and 152 cases without pancreatic ductal adenocarcinoma. Mod. Pathol. 2000; 16: 996 - 1006.

46. Adsay V, Logani S, Sarkar F, Crissman J, Vaikevicius V. Foamy gland pattern of pancreatic ductal adenocarcinoma: a deceptively benign appearing variant, Am.J. Surg. Pathol. 2000, 24: 493 504.

47. Wilentz RE, Goggins. M.et al. Genetic immunohistochemical, and clinical features of medullay carcinoma of the pancreas. A newly described and characterized entity. Am.j. Pathol. 2000, 15: 1641 - 1651. 
48. Lonardo F, Cubilla A1, Klimmsa DS. Microadenocarcinoma of the pancreas - morphologic pattern or pathologic entity. A revaluation of the original series. Am. J. Surg. Pathol. 1996. 20: $1385-1393$.

49. Molberg KH, Heffess C et al. Undifferentiated carcinoma with osteoclast like giant cells of the pancreas and periampullary region. Cancer. 1998, 82: 1279 - 1287.

50. Posen JA. Giant cell tumor of the pancreas of the osteoclastic type associated with a mucous secreting cystadenocarcinoma. Human Pathol 1981, 12. 944 - 947.

51. Trepeta RW, Mathur B, et al. Giant cell tumor (osteolclastoma) of the pancreas. A tumor of the epithelial origin. Cancer 1981, 48: 2022 - 2028.

52. Basturk 0, Zamboni et al. Intraductal and papillary variants of acinar cell carcinoma: a new addition to the challenging differential diagnosis of intraductal neoplasms. Am. J. Surg. Pathol. 2007,$31 ; 363-370$.

53. Hsuesh C, Kuo. TT. Acinar cell carcinoma of the pancreas. Report of two cases with complex histomorphological features causing diagnostic problems.Int. J. Pancreatol 1992, 12: 305 - 313.

54. Klimstra DS, Heffess CS, Oertel JE, Rosai J, Acinar cell carcinoma of the pancreas. A clinicopathologic study of 28 cases. Am. J. Surg. Pathol. 1992, 6: 815 - 837.

\section{AUTHORS:}

1. L. Veena Kumari

\section{PARTICULARS OF CONTRIBUTORS:}

1. Professor \& HOD, Department of Pathology, Mallareddy Medical College for Women, Suraram, Qutbullapur, Hyderabad.

FINANCIAL OR OTHER COMPETING INTERESTS: None

\section{NAME ADDRESS EMAIL ID OF THE} CORRESPONDING AUTHOR:

Dr. L. Veena Kumari, \# 4-73/12/1, Road No. 2, Bhulaxmi Nagar, Vanasthalipuram, Hyderabad-70.

E-mail: lveenakumari@yahoo.com

Date of Submission: 07/04/2015. Date of Peer Review: 09/04/2015. Date of Acceptance: 28/04/2015. Date of Publishing: 07/05/2015. 